\title{
A few clarifications on MIRIBEL
}

Matthew Faria ${ }^{1,2}$, Mattias Björnmalm ${ }^{1,3}$, Edmund J. Crampin ${ }^{2, *}$ and Frank Caruso ${ }^{1, *}$

${ }^{1}$ ARC Centre of Excellence in Convergent Bio-Nano Science and Technology, and the Department of Chemical Engineering, The University of Melbourne, Parkville, Victoria 3010, Australia

${ }^{2}$ ARC Centre of Excellence in Convergent Bio-Nano Science and Technology, Systems Biology Laboratory, School of Mathematics and Statistics and Melbourne School of Engineering, The University of Melbourne, Parkville, Victoria 3010, Australia

${ }^{3}$ Department of Materials, Department of Bioengineering and Institute of Biomedical Engineering, Imperial College London, London, SW7 2AZ, UK

*Corresponding authors. edmund.crampin@unimelb.edu.au, fcaruso@unimelb.edu.au

We are inspired by the responses ${ }^{1-4}$ to our recently proposed "reporting standards" for bio-nano research (Minimum Information Reporting in Bio-Nano Experimental Literature - MIRIBEL). ${ }^{5}$ However, we wish to clarify several points made in MIRIBEL and discuss a few concerns raised by the community, with a view toward encouraging uptake of the MIRIBEL standard and improving future research.

A guide, not a barrier: Our vision for MIRIBEL is that it serves as a guide, rather than a barrier to research. The MIRIBEL reporting standards should facilitate, and not stand in the way of conducting and communicating research. We envision consultation of the MIRIBEL checklist early in bio-nano projects to help make decisions on which directions to pursue. Because of the diverse backgrounds of people working in bio-nano science, a common consensus on which components should be reported is vital - both to aid communication and to prevent omission of components from published research, which may seem unimportant to researchers of one background, but are vital to those in another. We believe that every component on the MIRIBEL checklist deserves consideration, although which components will be characterized depends on the material, nature of the investigation, and facilities available. 
Improving reproducibility, quantification, and comparability: Some of the researchers surveyed did not agree that the MIRIBEL standard would facilitate these values, which was further underlined in some of the commentary. ${ }^{2}$ However in fields outside of bio-nano research, minimum information standards and reporting checklists have contributed strongly to reproducibility and quantitative research. Examples include the establishment of the microarray gene expression data society and the MIAME (Minimum Information about a Microarray Experiment) standard, ${ }^{6}$ which enabled the "omics" approaches that are ubiquitous in current research, and the MIRIAM (Minimum Information Requested in the Annotation of Biochemical Models) standard, ${ }^{7}$ which has enabled reproducibility through the exchange of models in computational biology. As bio-nano research becomes more quantitative, ${ }^{8,9}$ adopting the MIRIBEL approach will enable the same type of transformation. Our own recent work ${ }^{10}$ also demonstrates how the use of MIRIBEL components can turn a qualitative in vitro assay into a quantitative assay.

An aid to meta-analysis: Our hope is for the community as a whole to adopt MIRIBEL. However, even if only a subset uses it, MIRIBEL will facilitate review and meta-analysis. Papers that adopt MIRIBEL and implement the companion checklist are easier to survey, as consulting the checklist will inform which components are reported. This makes meta-analysis and review substantially easier to perform. Ideally, this information would be available in a searchable database; however, the community must first come to agreement about which information is of interest and importance. The following quote is as true today as it was over a decade ago: "By standardizing formats and ontologies, it becomes possible to use computers to mine a cumulative data set produced in hundreds of experiments performed around the world."11

Improving communication across the bio-nano community: Communication across the various fields and sub-fields of bio-nano researchers needs to be improved. Groups who create nano- and micro- structures and study their interactions with biological systems share many of the same goals and face similar difficult challenges, whether they use organic components, inorganic materials, or biological methods of synthesis. Realizing targeted and personalized medicine may require hybrid 
approaches, blending ideas and strategies across these domains. Additionally, we believe that the nano- "synthesis" focused communities can learn much from the nano-toxicological community, which has a longer history of standardization and quantification. ${ }^{12,13}$ We should forge deeper relationships, collaborate on projects, and share techniques across different areas of bio-nano science.

Clinical translation is not the only goal: While clinical translation is a major aim of many bio-nano studies, we recognize that it is far from the being the only worthwhile goal of bio-nano research. Even if we limit ourselves to application-focused research, bio-nano systems can lead to improved research tools such as scaffolds for cell growth ${ }^{14}$ or nucleic acid delivery systems ${ }^{15}$. Furthermore, the road to clinical translation is a (very!) long one. Not every new composition of material should be presented as (or expected to be) a revolutionary therapeutic. Published research spans projects from "a new material with interesting properties but no known applications" to "a clinical trial of a bio-nano-based therapeutic". Fundamental and exploratory should lay the groundwork for understanding bio-nano interactions and subsequent applications; however, standardization will facilitate this.

The MIRIBEL standard is a first, not a last, step. MIRIBEL is a proposal, not the end of the discussion. While we believe that following MIRIBEL will improve research, it will likely need modification, and we applaud and support the development of more specific standards - for instance, the recently suggested MIBNE (Minimum Information about Nanomaterial Biocorona Experiments) for biomolecular corona studies. ${ }^{16}$ However, it is vital for any standard - MIRIBEL or otherwise - that a community accepts it, followed by more formal adoption and ultimately journal enforcement. And as we say at the beginning, standards should be viewed as guides, not encumbering but aiding research and taking it to the next level.

\section{References}

(1) Leong, H. S. et al. On the issue of transparency and reproducibility in nanomedicine. Nat. Nanotechnol. 14, 902-902 (2019). 
(2) Lammers, T. \& Storm, G. Setting standards to promote progress in bio-nano science. Nat. Nanotechnol. 14, 626-626 (2019).

(3) Florindo, H. F., Madi, A. \& Satchi-Fainaro, R. Challenges in the implementation of MIRIBEL criteria on nanobiomed manuscripts. Nat. Nanotechnol. 14, 627-628 (2019).

(4) Voices from the community. Nat. Nanotechnol. 14, 625-625 (2019).

(5) Faria, M. et al. Minimum information reporting in bio-nano experimental literature. Nat. Nanotechnol. 13, 777-785 (2018).

(6) Brazma, A. et al. Minimum information about a microarray experiment (MIAME)-toward standards for microarray data. Nat. Genet. 29, 365-371 (2001).

(7) Novère, N. Le et al. Minimum information requested in the annotation of biochemical models (MIRIAM). Nat. Biotechnol. 23, 1509-1515 (2005).

(8) Gottstein, C., Wu, G., Wong, B. J. \& Zasadzinski, J. A. Precise quantification of nanoparticle internalization. ACS Nano 7, 4933-4945 (2013).

(9) Thomas, D. G. et al. ISD3: A particokinetic model for predicting the combined effects of particle sedimentation, diffusion and dissolution on cellular dosimetry for in vitro systems. Part. Fibre Toxicol. 15, 6 (2018).

(10) Faria, M. et al. Revisiting cell-particle association in vitro: A quantitative method to compare particle performance. J. Controlled Release 307, 355-367 (2019).

(11) Under the MIAME sun. Nat. Methods 3, 415 (2006).

(12) Hartmann, N. B., Ågerstrand, M., Lützhøft, H.-C. H. \& Baun, A. NanoCRED: A transparent framework to assess the regulatory adequacy of ecotoxicity data for nanomaterials relevance and reliability revisited. Nanolmpact 6, 81-89 (2017).

(13) Guggenheim, E. J. et al. Refining in vitro models for nanomaterial exposure to cells and tissues. Nanolmpact 10, 121-142 (2018).

(14) Alegret, N., Dominguez-Alfaro, A. \& Mecerreyes, D. 3D Scaffolds based on conductive polymers for biomedical applications. Biomacromolecules 20, 73-89 (2019). 
(15) Ni, R., Feng, R. \& Chau, Y. Synthetic approaches for nucleic acid delivery: Choosing the right carriers. Life 9, 59 (2019).

(16) Chetwynd, A. J., Wheeler, K. E. \& Lynch, I. Nano Today 28, 100758 (2019). 